\title{
Why the metabolism field risks missing out on the $\mathrm{Al}$ revolution
}

\author{
A collaborative effort is required by individual scientists, research institutes and funding organizations towards \\ the curation of high-quality, diverse metabolic data for the metabolism community to leverage the full potential \\ of artificial intelligence.
}

W e are witnessing an era in which artificial intelligence (AI) has moved from theoretical science, or science fiction, into real-world application, most notably in the fields of robotics, electronics, communications, engineering and image processing. However, AI's application in the biomedical sciences has lagged behind its application in information technology. Nonetheless, the biomedical sciences field is catching up, not only in the number of AI applications but also in terms of AI-related publications and funding. Over the years, the biomedical and life sciences have seen a slow but steady increase in research articles focused on AI-assisted applications in areas such as drug discovery, imaging, diagnostics, genetics, psychology and cognitive science. Encouraging demonstrations of the application of machine learning (ML) in clinics for accurate prediction or diagnosis of diseases-including cancer, cardiovascular diseases, kidney diseases or genetic disorders-have fuelled collective optimism that AI may have the potential to revolutionize the clinical sciences. However, basic metabolic research and research on metabolic diseases have not advanced through application of AI as much as other areas of the biomedical or life sciences, such as neuroscience and genomics. This lag prompts questions of why AI is important for the field of metabolism and what can be done collectively to ensure that the wider metabolism field does not miss out on the AI revolution.

One reason why AI in the metabolism field has fallen behind is the paucity of highquality datasets, which are crucial for the successful application of ML algorithms and platforms. Other problems are incomplete metadata, technical mistakes during data acquisition and processing, and the lack of both experimental and technical quality controls. Pre-cleaning and appropriate normalization of data by statisticians are extremely important to ensure that ML algorithms work effectively. The dearth of data scientists in what traditionally used to be 'wet' biology labs and the poor statistical training of biologists are two likely reasons

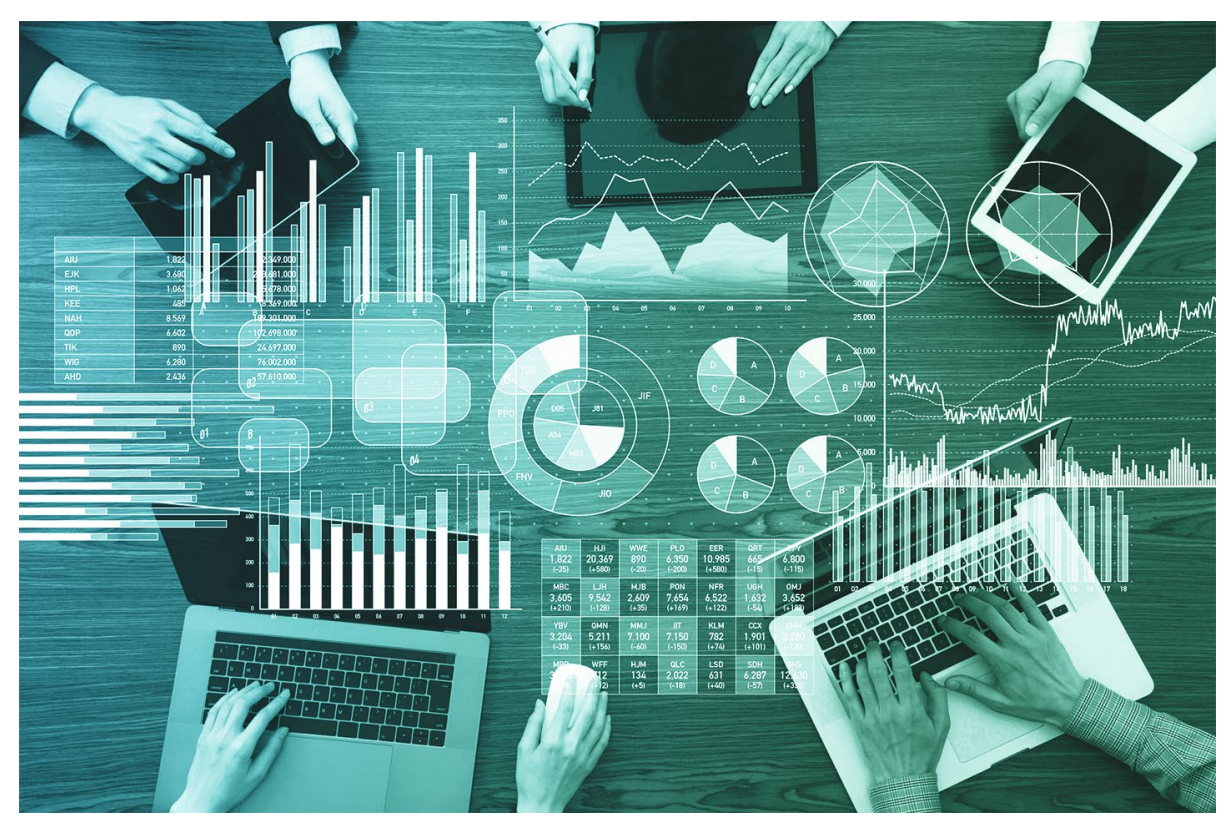

Credit: Metamorworks / iStock / Getty Images Plus / Getty

why the quality of metabolic datasets is often insufficient for ML applications.

What can be done to solve this problem? Given the massive amounts of data being generated each day, the scientific community must ensure that data are acquired, processed, cleaned and documented to the highest standards so that they can be effectively used by AI algorithms and platforms to yield meaningful results. A central challenge for the metabolism community remains the integration of large datasets obtained by different platforms and labs. Therefore, curation of high-quality, diverse data requires a collective effort at all levels, including individual researchers, labs, institutes and, not least, publishers. Universities, scientific institutes and funding bodies should regulate and mandate proper data management and storage, ideally in script- or algorithm-readable formats. Some institutes (such as École Polytechnique Fédérale de Lausanne) and funding bodies (such as the European Research Council) are already leading the way: The European Research Council, for example, mandates an open-research data-management plan with the goal of making project data 'findable, accessible, interoperable and reusable (FAIR)', representing a step in the right direction for data accountability and potential use in AI and ML applications.

AI research and applications are rapidly increasing worldwide. According to data from the Springer Insight platform, the ten countries funding the largest number of metabolism-related grants from 2016 to 2028 are the United States $(4,870)$, China (4,864), Japan (1,516), Canada (722), the United Kingdom (701), Brazil (673), Germany (438), Belgium (409), Russia (319) and Sweden (258). With regards to ML funding in the same time period, the United States is currently leading (4,700 active grants), followed by the United Kingdom $(1,329)$, China $(1,187)$, Canada $(646)$, Japan (570), Belgium (456), Germany (358), Sweden (281), Russia (206) and Switzerland (203). China and the United States may thus have a competitive advantage in exploiting AI-assisted tools in the areas of metabolic research over the next decade. 
However, China certainly has ambitions to close the gap with the United States: the New Generation Artificial Intelligence Development Plan, announced in July 2017 by the Chinese State Council, lays out a strategy for China to become the world leader in AI by 2030.

Metabolism and metabolic diseases are complex and multifactorial. It is becoming increasingly clear that the answers regarding the fundamental mechanisms, pathways and therapies for metabolic disorders will involve the study of related research disciplines-such as gut metabolism, immunometabolism, cancer metabolism, stem cell metabolism and genetics-and will call for integrative 'omics' approaches, such as proteomics, transcriptomics, metabolomics, genomics, epigenetics and others. Adding to this complexity are the data generated from numerous transgenic-mouse models and human population studies. With the volume of data increasing daily, and omics approaches becoming routinely applied, it will be particularly important that each and every dataset generated be both usable and reusable. Such curation would decrease data pollution and enable the metabolism field to take full advantage of the power of AI for the benefit of science and human health.

Published online: 14 October 2019 https://doi.org/10.1038/s42255-019-0133-9 